\title{
Mechanical design and analysis of a novel variable stiffness actuator with symmetrical pivot adjustment
}

(C) The Author(s) 2021. This article is published with open access at link.springer.com and journal.hep.com.cn

\begin{abstract}
The safety of human-robot interaction is an essential requirement for designing collaborative robotics. Thus, this paper aims to design a novel variable stiffness actuator (VSA) that can provide safer physical humanrobot interaction for collaborative robotics. VSA follows the idea of modular design, mainly including a variable stiffness module and a drive module. The variable stiffness module transmits the motion from the drive module in a roundabout manner, making the modularization of VSA possible. As the key component of the variable stiffness module, a stiffness adjustment mechanism with a symmetrical structure is applied to change the positions of a pair of pivots in two levers linearly and simultaneously, which can eliminate the additional bending moment caused by the asymmetric structure. The design of the doubledeck grooves in the lever allows the pivot to move freely in the groove, avoiding the geometric constraint between the parts. Consequently, the VSA stiffness can change from zero to infinity as the pivot moves from one end of the groove to the other. To facilitate building a manipulator in the future, an expandable electrical system with a distributed structure is also proposed. Stiffness calibration and control experiments are performed to evaluate the physical performance of the designed VSA. Experiment results show that the VSA stiffness is close to the theoretical design stiffness. Furthermore, the VSA with a proportional-derivative feedback plus feedforward controller exhibits a fast response for stiffness regulation and a good performance for position tracking.
\end{abstract}

Keywords variable stiffness actuator, variable stiffness module, drive module, symmetrical structure, doubledeck grooves, expandable electrical system

Received March 23, 2021; accepted May 26, 2021

Yiwei LIU ( $₫)$, Shipeng CUI, Yongjun SUN

State Key Laboratory of Robotics and System, Harbin Institute of Technology, Harbin 150001, China

E-mail: lyw@hit.edu.cn

\section{Introduction}

Traditional manipulators are mostly used in industrial production, working in a structured environment on a given trajectory to complete specific tasks. To ensure excellent tracking performance and safety, manipulators generally adopt the design scheme of rigid mechanical structure and active compliance control such as position/force [1,2] or impedance/admittance control $[3,4]$. However, rigid manipulators based on active compliance control still present stiffness [5] when suffering sudden impacts or handling contact transients due to the control delay, which can cause damage to itself or surrounding environments. To address these issues, the flexible actuator with inherent compliance has received substantial attention in recent years and has been widely applied in robotics [6-8]. The flexible actuator is equipped with an elastic element that can buffer impact and provide additional time for the control system to implement effective countermeasures $[9,10]$.

As a typical representative of the flexible actuator, the variable stiffness actuator (VSA) [11-13] aims to imitate the characteristics in dynamics and kinematics of a biosystem. Same as series elastic actuator [14-16], VSA can increase peak power output [17] and reduce energy consumption [18] by storing and releasing the potential energy stored in the elastic element. In addition to the advantages of series elastic actuator, VSA can increase the adaptability of manipulators by actively adjusting its own stiffness to meet different task requirements for improving performances [19] and exhibits advantages in terms of energy efficiency [20-22].

According to structural form, VSAs are classified into two categories, namely, antagonistic VSA and serial VSA. Antagonistic VSA [20,23-26] is inspired by the skeletal system of living creatures with the antagonistic arrangement of muscles, usually possessing two motors. Each motor is connected to a nonlinear elastic element to drive the actuator output antagonistically. The position 
and stiffness of antagonistic VSA are simultaneously controlled by two same motors: Motors move in the opposite direction for stiffness adjustment and work in the same direction for changing actuator position. Despite the simple structure for easy engineering implementation, the main limitation of antagonistic VSA is the synchronous control of position and stiffness that leads to control complexity and high energy consumption. Compared with antagonistic VSA, the position and stiffness of serial VSA are controlled by two different motors: One principal motor changes position, and an auxiliary motor adjusts stiffness independently. The representative serial VSAs, such as MACCEPA 2.0 [27], DLR VS-joint [28], FSJ [29], and $\mathrm{S}^{3} \mathrm{VSA}$ [30] adjust stiffness by changing the pretension or preload on the elastic element with the stiffness motor. The main limitation of these serial VSAs is rooted in the higher requirements on the driving capacity of the stiffness motor. Other serial VSAs adjust stiffness by changing the transmission ratio between the actuator output and the elastic element. Among these serial VSAs, the ones based on the lever principle with variable pivot [31-37] have a theoretical stiffness adjustment range from zero to infinity. Furthermore, as described in Refs. [21,38-40], the output stiffness of these actuators can be regulated without energy injection or extraction from the elastic element, which means that all the energy from motors work on the load. Therefore, compared with the other counterparts, VSAs based on the lever principle with variable pivot dominate in terms of stiffness adjustment range and energy efficiency. Nevertheless, the existing VSAs based on the lever principle with variable pivot $[31,32,36,37]$ mostly have the asymmetric structure of a single pivot and lever that will cause stress concentration, bring additional bending moment, and decrease mechanical reliability. Though the SVSA-II [41] based on a symmetrical adjustable pivot lever mechanism is proposed, the stiffness adjustment mechanism is designed together with the VSA as a whole rather than being designed as a separate module, which makes the VSA difficult to maintain. A leaf-spring-based stiffness adjustment mechanism following the modular design is presented in Ref. [42], but the stiffness can only be adjusted manually. In Ref. [43], a new configuration synthesis method to design modular stiffness adjustment mechanism based on guide-bar mechanisms through the addition of linear springs and the use of length-adjustable links is proposed. However, the stiffness adjustment mechanism designed is still in the modeling stage, rather than the prototype.

To address these issues, this paper highlights research in the design of a modular serial VSA with symmetrical pivot adjustment. Our contributions can be summarized as follows. 1) A symmetrical stiffness adjustment mechanism is developed to change the positions of a pair of pivots. Compared with other VSAs based on the lever principle with variable pivot, the symmetrical stiffness adjustment mechanism of the designed VSA can eliminate the bending moment caused by the asymmetric structure and enhance the reliability and stability of the internal structure. 2) The stiffness of the designed VSA can change from zero to infinity by virtue of the doubledeck grooves design in the lever that enables the pivot to move freely. 3) A roundabout manner of transmitting motion that can realize modularization of the VSA is presented.

The remainder of this paper is organized as follows. The principle of stiffness adjustment is introduced, together with the characteristics of VSA employing this principle in Section 2. The physical implementation of the designed VSA is presented in Section 3. The stiffness calibration and stiffness adjustment experiments are carried out in Section 4, and several conclusions are drawn in Section 5.

\section{Schematic design of stiffness adjustment}

Based on the lever principle, a stiffness adjustment scheme with symmetrical variable pivot is proposed. The corresponding characteristics of this stiffness adjustment are also analyzed. The proposed scheme theoretically has a simple structure for easy engineering implementation.

\subsection{Principle of stiffness adjustment}

The schematic diagram of the stiffness adjustment principle, which presents a simplified model of the variable stiffness module, is shown in Fig. 1. Two sets of levers, pivots, and linear springs are arranged symmetrically on the variable stiffness module. The input link represents the input of the variable stiffness module that is connected to the output of the drive module and transmits the motion and torque of the drive module. The output link can be regarded as the output of the variable stiffness module that is connected to the output of the VSA. Its center coincides with that of the variable stiffness module. Two linear springs assembled on the input link are antagonistically connected to one end of the lever with a suitable pretension at the spring point. The

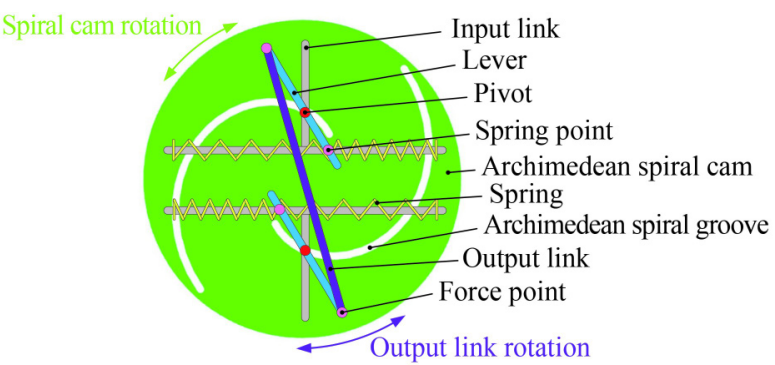

Fig. 1 Design principle of variable stiffness module. 
other end of the lever is connected to the output link at the force point. The pivot is inserted into the grooves of the lever, input link, and Archimedean spiral cam. The spring axis is perpendicular to the linear groove of the input link. The Archimedean spiral cam is used to convert its rotational motion into translational motion of the pivot through the linear groove and spiral groove of the input link and Archimedes spiral cam, respectively. If load force is exerted on the VSA, the output link compels the lever rotating around the pivot to change the position of the spring point, resulting in the flexible deformation angle of the VSA. When the Archimedean spiral cam rotates relative to the link driven by the stiffness motor, the pivot can slide on the linear groove of the input link from the spring point to the force point, changing the lever ratio for stiffness adjustment.

The symmetrical design of the variable ratio lever principle for stiffness adjustment proposed in this paper introduces two advantages:

1) It can increase load capacity of the VSA in the limited structural space.

2) It can eliminate the additional bending moment caused by the asymmetric structure and improve the reliability and stability of the VSA.

\subsection{Characteristics of the VSA}

Figures 2(a) and 2(b) show the schematic diagrams of the internal structure and corresponding force analysis of the variable stiffness module, when load force is applied on the output link. The symmetry axis of the variable stiffness module is represented by the dot dash line, and only half of the structure is presented in Fig. 2.

In Fig. 2(a), $a$ is the vertical distance from the pivot to the spring axis; $b$ is the distance between the spring axis and the symmetrical center line of the variable stiffness module; $R$ is half the total length of the output link, i.e., the distance between the force point and the center of the variable stiffness module; $\Delta x$ is the deformation of the spring; $l_{1}$ and $l_{2}$ are the distances from the pivot to the spring point and the force point, respectively; $\varphi$ is the angle between the input link and the output link that represents the flexible deformation angle of the VSA; $\gamma$ is the angle between the lever and the linear groove of the input link; and $A$ is the angle between the lever and the output link. In Fig. 2(b), $F_{1}$ is the load force applied at the force point; $F_{1 \mathrm{t}}$ is decomposed along the direction perpendicular to the lever; $F_{\mathrm{p}}$ is the force applied on the lever from the pivot; $F_{\mathrm{pa}}$ is decomposed along the direction perpendicular to the spring axis; $F_{\mathrm{s}}$ is the spring force; $F_{\mathrm{st}}$ is decomposed along the direction perpendicular to the lever; and $F_{\mathrm{e}}$ is the elastic force of the VSA generated at the force point in the opposite direction of load force $F_{1}$.

According to Fig. 2(a), the deformation of the spring $\Delta x$ can be expressed as

$$
\Delta x=a \tan \gamma .
$$

Then, the spring force generated by the spring can be computed as

$$
F_{\mathrm{s}}=2 k \Delta x=2 k a \tan \gamma,
$$

where $k$ is the spring stiffness.

The component force $F_{\text {st }}$ of spring force $F_{\mathrm{s}}$ is given by

$$
F_{\mathrm{st}}=F_{\mathrm{s}} \cos \gamma=2 k a \tan \gamma \cos \gamma=2 k a \sin \gamma .
$$

According to the lever principle, $F_{1 \mathrm{t}}$ can be calculated as

$$
F_{\mathrm{lt}}=\frac{F_{\mathrm{st}} l_{1}}{l_{2}}=\frac{2 k a l_{1} \sin \gamma}{l_{2}} .
$$

Thus, load force $F_{1}$ can be computed as

$$
F_{1}=\frac{F_{1 \mathrm{t}}}{\cos \alpha}=\frac{2 k a l_{1} \sin \gamma}{l_{2} \cos \alpha} .
$$

According to Fig. 2(a), the following geometrical relations can be obtained:

$$
\left\{\begin{array}{l}
l_{2} \cos \alpha=R-(a+b) \cos \varphi, \\
l_{1} \sin \gamma=\Delta x=a \tan \gamma=\frac{a R \sin \varphi}{R \cos \varphi-(a+b)} .
\end{array}\right.
$$

Combining Eqs. (5) and (6), the load torque exerted on the output link $\left(\tau_{1}\right)$ can be expressed as

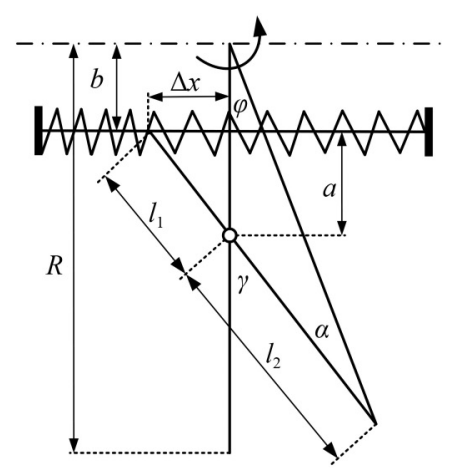

(a)

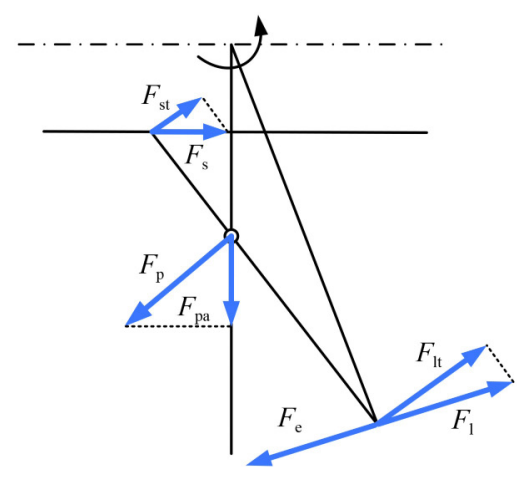

(b)

Fig. 2 Schematic of the stiffness adjustment principle. (a) Geometrical analysis; (b) force analysis. 


$$
\tau_{1}=2 F_{1} R=\frac{4 k a^{2} R^{2} \sin \varphi}{[R \cos \varphi-(a+b)][R-(a+b) \cos \varphi]} .
$$

Moreover, the stored elastic energy $(E)$ from the compression springs is given by

$$
E=2 k \Delta x^{2}=\frac{2 k a^{2} R^{2} \sin ^{2} \varphi}{[R \cos \varphi-(a+b)]^{2}} .
$$

According to the stiffness definition, the stiffness of the $\operatorname{VSA}(K)$ can be calculated as

$$
\begin{aligned}
K & =-\frac{\partial \tau_{\mathrm{e}}}{\partial \varphi}=\frac{\partial \tau_{1}}{\partial \varphi} \\
& =4 k a^{2} R^{2} \frac{[R \cos \varphi-(a+b)]^{2}+R \sin ^{2} \varphi[R-(a+b) \cos \varphi]}{[R \cos \varphi-(a+b)]^{2}[R-(a+b) \cos \varphi]^{2}},
\end{aligned}
$$

where $\tau_{\mathrm{e}}$ is the elastic torque of the variable stiffness module. Equation (9) shows that the stiffness of the VSA can be adjusted by $a$, i.e., the position of the pivot.

The force $F_{\mathrm{p}}$ applied on the lever from the pivot can be written as

$$
F_{\mathrm{p}}=\frac{F_{\mathrm{st}}\left(l_{1}+l_{2}\right)}{l_{2}} \text {. }
$$

Thus, the component force $F_{\mathrm{pa}}$ of force $F_{\mathrm{p}}$ is given by

$$
F_{\mathrm{pa}}=F_{\mathrm{p}} \sin \gamma=\frac{F_{\mathrm{st}}\left(l_{1}+l_{2}\right) \sin \gamma}{l_{2}} .
$$

To select the appropriate stiffness motor, the force analysis of the Archimedean spiral cam is presented. The schematic diagrams of the Archimedean spiral cam and its force analysis are shown in Fig. 3.

Figure 3(a) shows that the Archimedean spiral line in the rectangular coordinate can be expressed by the following equations:

$$
\left\{\begin{array}{l}
x=(d+c \rho) \cos \rho, \\
y=(d+c \rho) \sin \rho,
\end{array}\right.
$$

where $\rho$ is the polar angle of point $P(x, y)$ on the Archimedean spiral line, and $d$ is the polar radius when $\rho=0$.

If $d=0$, the initial point of the Archimedean spiral line coincides with the origin of the coordinate. The distance between two successive turnings is $2 \pi c$. The curvature $(S)$ of the Archimedean spiral line at $P(x, y)$ can be calculated as

$$
S=\frac{\mathrm{d} y}{\mathrm{~d} x}=\frac{d \cos \rho+c \sin \rho+c \rho \cos \rho}{-d \sin \rho+c \cos \rho-c \rho \sin \rho} .
$$

Force $f_{\mathrm{p}}$ applied by the pivot on the Archimedean spiral cam is equal but opposite to force $F_{\mathrm{p}}$ on the lever, i.e., $f_{\mathrm{p}}=-F_{\mathrm{p}} \cdot f_{\mathrm{pa}}$ is decomposed of $f_{\mathrm{p}}$ along the direction perpendicular to the spring axis. Figure 3(b) shows that $f_{\text {pat }}$ is decomposed of $f_{\text {pa }}$ along the direction perpendicular to the tangent of the Archimedean spiral line. $r$ is the arm of the force $f_{\text {pat }}$ relative to the coordinate origin. $\beta$ is the angle between the force $f_{\mathrm{pa}}$ and the tangent of the Archimedean spiral line. It can be formulated as

$$
\beta=\arctan \frac{S-S_{1}}{1+S S_{1}},
$$

where $S_{1}$ is the slope between point $P(x, y)$ and the coordinate origin, i.e., $S_{1}=y / x$.

According to Fig. 3, component force $f_{\text {pat }}$ can be expressed as

$$
f_{\mathrm{pat}}=f_{\mathrm{pa}} \sin \beta \text {. }
$$

The torque applied by the pivot on the Archimedean spiral cam is given by

$$
\tau_{\mathrm{p}}=f_{\text {pat }}(a+b) \cos \beta=f_{\mathrm{pa}}(a+b) \sin \beta \cos \beta .
$$

The selection of the stiffness motor depends on the resistance torque exerted by the stiffness motor that keeps the Archimedean spiral cam stationary relative to the input link that can maintain the position of the pivot. Resistance torque $\tau_{\mathrm{r}}$ can be calculated as

$$
\begin{aligned}
\tau_{\mathrm{r}} & =2 \tau_{\mathrm{p}}=2 f_{\mathrm{pa}}(a+b) \sin \beta \cos \beta \\
& =(a+b) \sin 2 \beta F_{\mathrm{st}}\left(l_{1}+l_{2}\right) \sin \gamma / l_{2} \\
& =2 k a(a+b) \sin 2 \beta \sin ^{2} \gamma\left(l_{1}+l_{2}\right) / l_{2} \\
& =\frac{2 k a R^{2}(a+b) \sin 2 \beta \sin ^{2} \varphi(R \cos \varphi-b)}{(R \cos \varphi-a-b)\left[R^{2}+(a+b)^{2}-2(a+b) R \cos \varphi\right]} .
\end{aligned}
$$

According to the characteristics of the drive module designed in Section 3, specific values of the dimension parameters in the variable stiffness module are listed

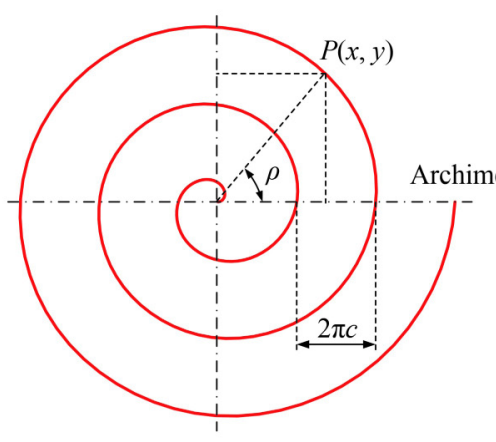

(a)

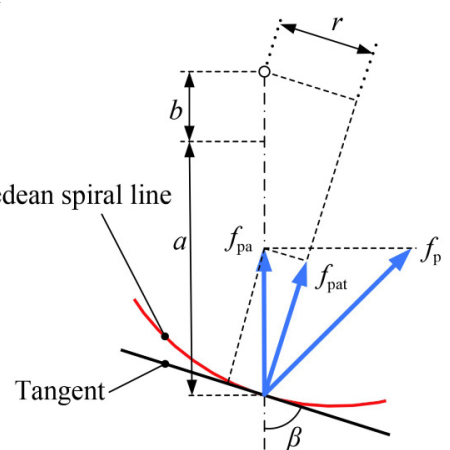

(b)

Fig. 3 Analysis of Archimedean spiral cam. (a) Archimedean spiral line; (b) force analysis. 
below: $b=16 \mathrm{~mm}, R=34 \mathrm{~mm}, d=0 \mathrm{~mm}, k=6100 \mathrm{~N} / \mathrm{m}$, $c=8.0212 \mathrm{~mm}$.

Thus, the movable range of the pivot is from 0 to 18 $\mathrm{mm}$. The effective length of the lever increases with the increase of flexible deformation angle $\varphi$. As shown in Fig. 2, the following equations can be obtained:

$$
\left\{\begin{aligned}
l_{1} & =\sqrt{(\Delta x)^{2}+a^{2}}=\sqrt{(a \tan \gamma)^{2}+a^{2}} \\
& =\sqrt{\left(a \frac{R \sin \varphi}{R \cos \varphi-a-b}\right)^{2}+a^{2}}, \\
l_{2} & =\sqrt{(R \sin \varphi)^{2}+(R-a-b)^{2}} .
\end{aligned}\right.
$$

The maximum flexible deformation angle is related to the pivot position because the total length of the lever is limited, i.e., $l_{1}+l_{2} \leqslant l$. where $l=21 \mathrm{~mm}$. According to Eq. (18), this relation can be expressed by Fig. 4. If pivot position $a$ is 0 , the value of the maximum flexible deformation angle is $26.81^{\circ}$. Maximum deformation $\varphi_{\max }$

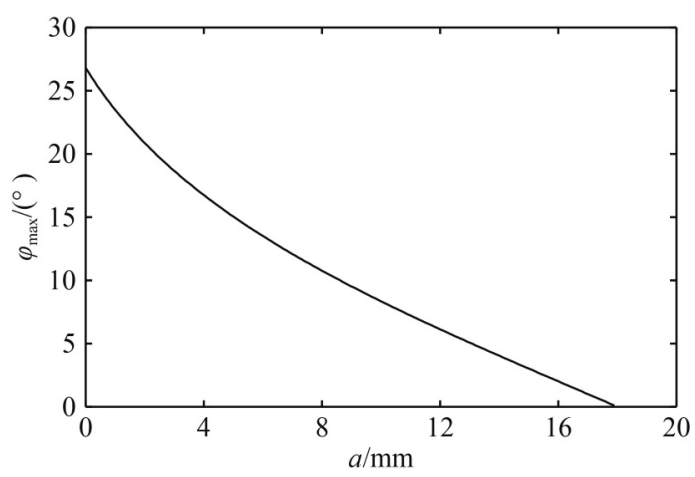

Fig. 4 Maximum flexible deformation angle versus pivot position.

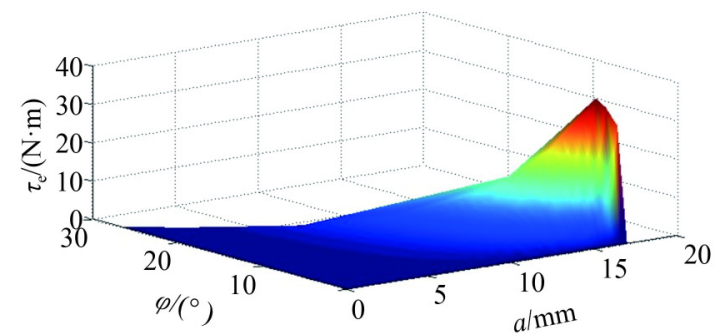

(a)

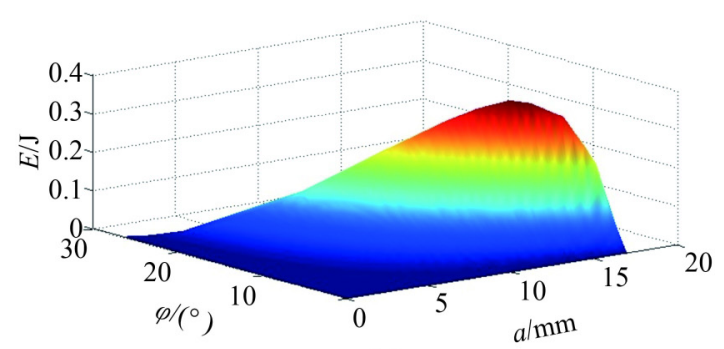

(c) substantially decreases for increasing values of pivot position.

Equations (7)-(9) and (17) illustrate that pivot position $a$ and flexible deformation angle $\varphi$ can affect the characteristics of the VSA. The results of the theoretical elastic torque, VSA stiffness, stored elastic energy, and resistance torque of the stiffness motor when the pivot position is changed from 0 to $17 \mathrm{~mm}$ and the deformation angle is changed from $0^{\circ}$ to $26.81^{\circ}$ are presented in Fig. 5 .

Figure 5 shows that elastic torque, VSA stiffness, stored elastic energy, and resistance torque have similar variation trends. If the pivot position is fixed, they increase with the increase of the flexible deformation angle. If the flexible deformation angle is fixed, they increase slightly when $a$ is changed from 0 to $13 \mathrm{~mm}$, and they increase dramatically to the peak value when the pivot is considerably close to $17 \mathrm{~mm}$. When the pivot position is at $17 \mathrm{~mm}$, the maximum flexible deformation angle is $1.21^{\circ}$. In this case, the values of the elastic torque, VSA stiffness, and stored elastic energy reach their maximum values of $36 \mathrm{~N} \cdot \mathrm{m}, 1708 \mathrm{~N} \cdot \mathrm{m} / \mathrm{rad}$, and $0.385 \mathrm{~J}$, respectively. According to Fig. 5(d), when the elastic torque of the VSA is $36 \mathrm{~N} \cdot \mathrm{m}$, the stiffness motor is required to provide a torque of $3.84 \mathrm{~N} \cdot \mathrm{m}$ to maintain the pivot position. Theoretically, VSA stiffness and elastic torque increase to infinity when the pivot is close to the force point. However, due to the limitation of the motor driving ability, the elastic torque of the VSA is not more than the maximum torque that the drive module can provide.

\section{System design of the VSA}

The modular design, which can strengthen

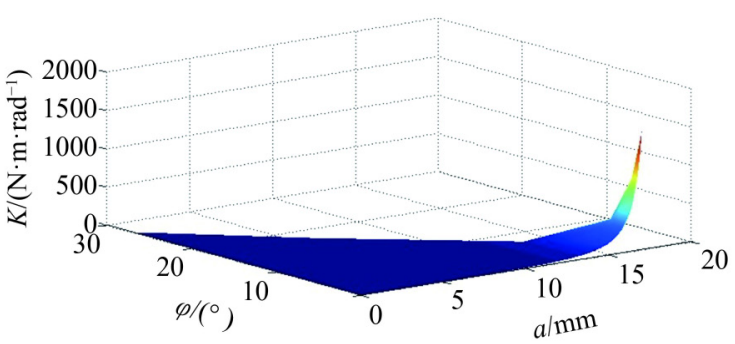

(b)

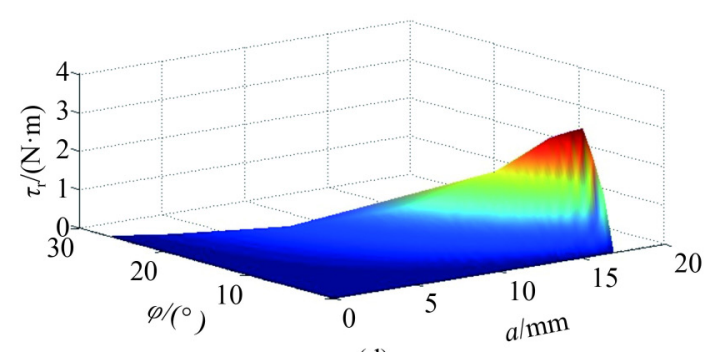

(d)

Fig. 5 Theoretical characteristics of VSA: (a) elastic torque, (b) stiffness, (c) elastic energy, and (d) resistance torque. 
interchangeability of parts and reduce development costs, has become a trend in the development of actuators or manipulators. At present, traditional actuators are many, but VSAs are less, developed by modular design. Therefore, this paper adopts the idea of modular design from mechanical structure and electrical system to develop the VSA. The underlying idea of modular design is that a rigid actuator can be converted into a VSA by installing the variable stiffness module developed in this paper. Based on the stiffness adjustment scheme proposed in the previous section, the mechanical structure of the VSA and the corresponding prototype designed in this paper is shown in Fig. 6.

Figure 6 shows that the VSA integrating the systems of the drive, transmission, and sensors is mainly composed of drive module and variable stiffness module. The two modules are connected by screws and can work independently. The maximum length and maximum diameter of the VSA are 156 and $104 \mathrm{~mm}$, respectively. To reduce the weight, almost all the mechanical parts are made of aluminum alloy 7A09 under the premise of ensuring strength requirements, resulting in a lighter VSA with a total mass of $2.46 \mathrm{~kg}$. The VSA designed in this paper is completely suitable to be employed as a module to build the manipulator owing to its compact volume, light weight, and high power.

\subsection{Design of the drive module}

The CAD model of the drive module is shown in Fig. 7. The drive module is composed of a brake mechanism and a drive mechanism. The drive mechanism mainly includes actuator base, actuator output, principal motor, harmonic reducer, motor shaft, and output shaft. The brake mechanism mainly includes electromagnet, springs, armature, brake plate, and friction disks.

The drive mechanism provides power for the motion of the VSA. A brushless DC motor 72-S developed by the Harbin Institute of Technology, China (HIT) is selected as the principal motor. The rated torque and speed of this motor are $0.36 \mathrm{~N} \cdot \mathrm{m}$ and $3600 \mathrm{r} / \mathrm{min}$, respectively. The motor stator and rotor are attached to the actuator base and motor shaft by adhesive, respectively. To make the drive mechanism as compact as possible, a cup type harmonic reducer CSD-20-160-2A-GR of Harmonic Drive Company is selected to transfer the motion of the

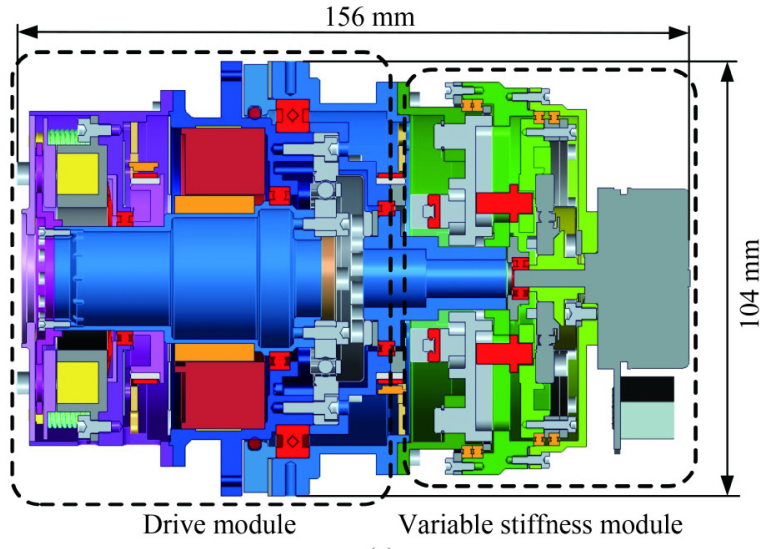

(a)

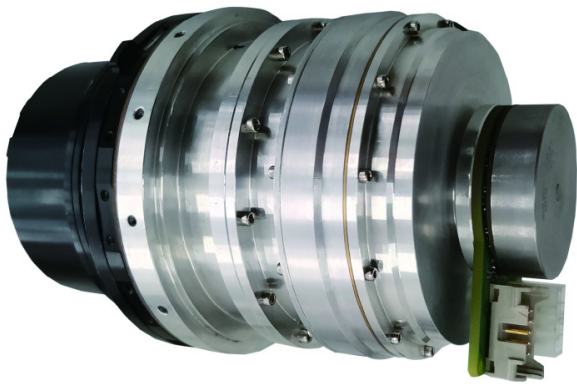

(b)

Fig. 6 Design of the VSA. (a) CAD model of the VSA; (b) prototype of the VSA.

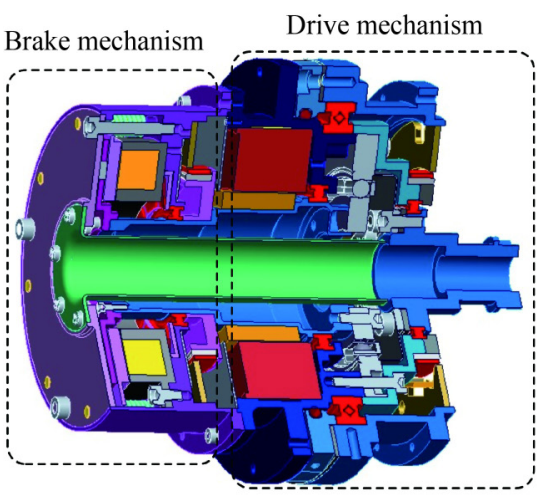

(a)

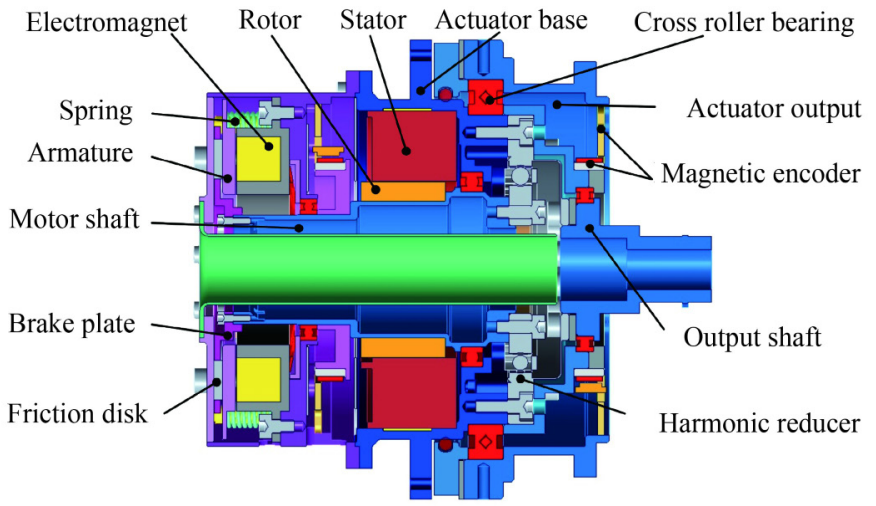

(b)

Fig. 7 CAD model of the drive module. (a) Section view; (b) side view. 
principal motor. The wave generator, flex spline, and circular spline of the harmonic reducer are connected to the motor shaft, actuator base, and output shaft by screws, respectively. The drive mechanism employs a cross roller bearing RA7008C of THK Company to achieve an accurate, stable rotation between the actuator output and the actuator base replacing a pair of angular contact ball bearings, which can make the actuator more compact. Moreover, the drive mechanism is equipped with two absolute magnetic encoders developed by HIT $[44,45]$ to measure the positions of the motor shaft and actuator output relative to the actuator base. The magnetic encoder with a 16-bit resolution and an accuracy of $20^{\prime \prime}$ mainly consists of a code disk and a signal processing part.

To maintain the current position of the VSA when power is off, the drive module integrates a brake mechanism that can brake the drive module in a frictional manner. The shell of the brake mechanism and the brake plate are connected to the actuator base and the motor shaft by screws, respectively. Eight friction disks made of asbestos are evenly embedded on the brake plate. When the drive module is working, the armature is pulled by the energized electromagnet against the spring force away from the friction disks, and then the motor shaft can rotate freely. When power is off, the motor shaft stops because the spring force presses the armature on the friction disks.

If a torque sensor or a flange replaces the output shaft of the drive mechanism and connects to the actuator output, the drive module can be converted into a common rigid actuator.

\subsection{Design of the variable stiffness module}

According to the design principle of the variable stiffness scheme proposed in Section 2, the CAD model of the variable stiffness module designed in this paper is shown in Fig. 8. The corresponding prototype is shown in Fig. 9. The variable stiffness module is composed of an elastic force transmission mechanism and a stiffness adjustment mechanism. The elastic force transmission mechanism mainly includes the output of the variable stiffness module, thin section bearings, linear springs, linear guide rails, sliders, spring bases, and levers. The stiffness adjustment mechanism mainly includes the input of the variable stiffness module, Archimedean spiral cam, pivots, thin section bearings, connector, motor base, and stiffness motor.

Figure 6 shows that the output shaft of the drive module is connected to the input of the variable stiffness module by flat keys, and the actuator output is connected to the output of the variable stiffness module by screws. Figure

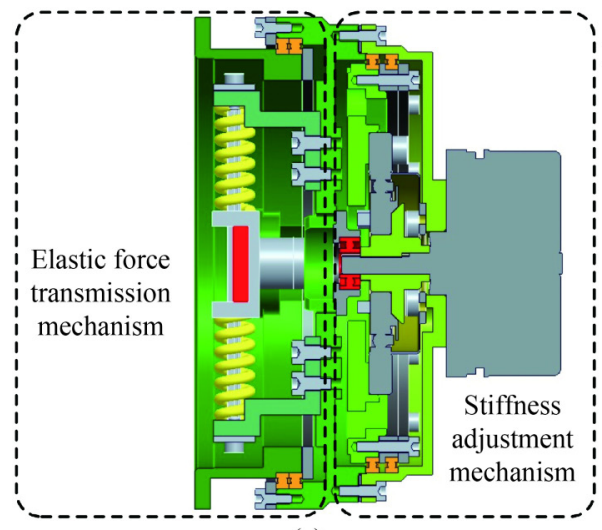

(a)

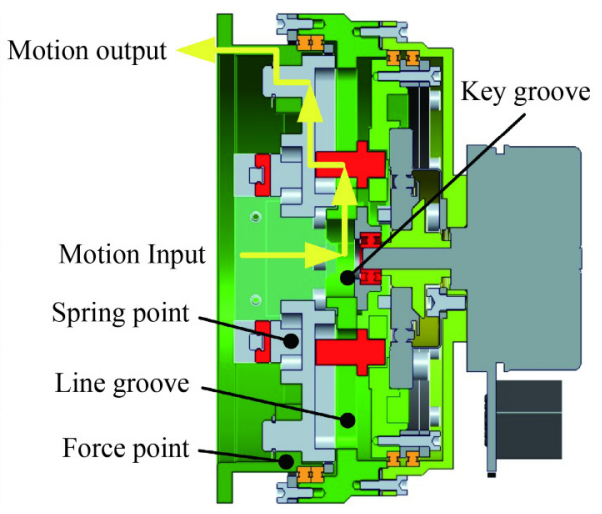

(b)

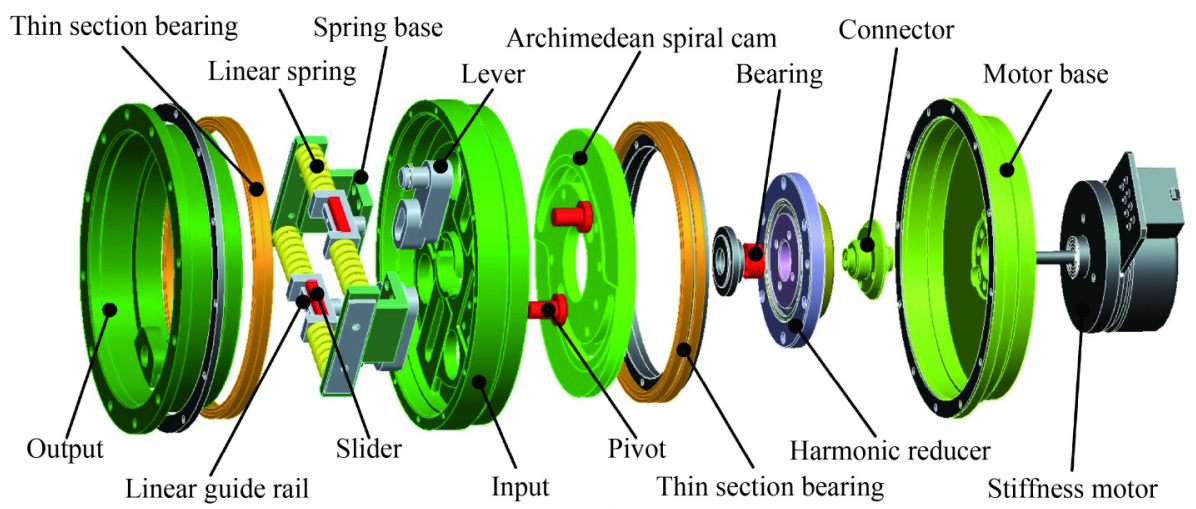

(c)

Fig. 8 CAD model of the variable stiffness module. (a) Side view 1; (b) side view 2; and (c) explosive view. 

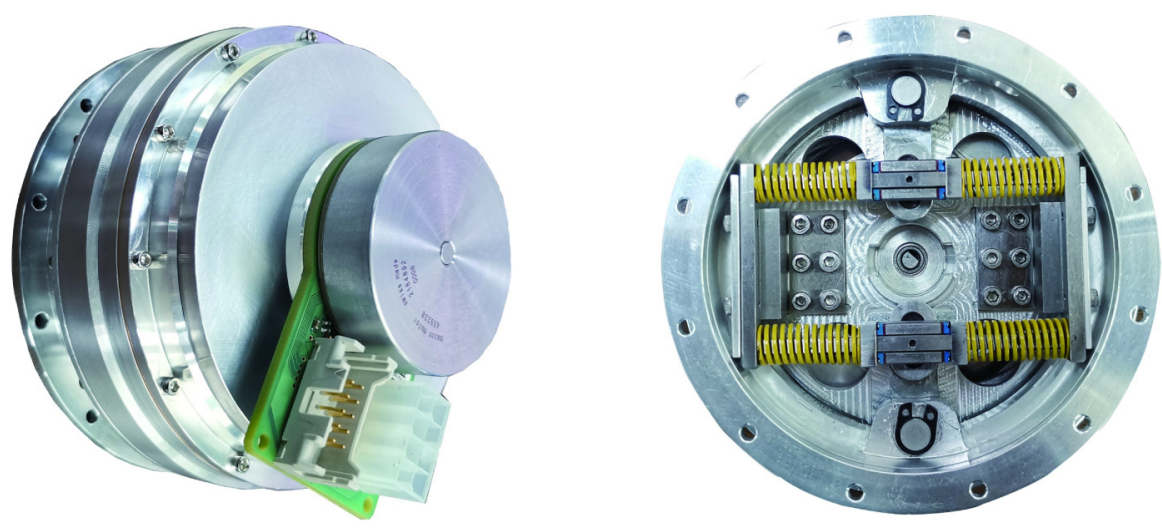

Fig. 9 Prototype of the variable stiffness module.

8(b) shows that the motion of the drive module is transmitted to the actuator output of the drive module in a roundabout manner through the input of the variable stiffness module, pivots, levers, and the output of the variable stiffness module. Transmitting motion in the roundabout manner has two main advantages: 1) The modular design of the stiffness adjustment mechanism can be realized, and then a common rigid actuator can be transformed into a VSA by mounting the variable stiffness module; 2) the distance from the actuator output to the actuator base is not increased due to the mounting of the variable stiffness module; hence, the overturning moment applied to the rotating part (bearing) of the original rigid actuator remains unchanged.

A pair of linear guide rails inserted with linear springs is arranged symmetrically on the spring bases fixed on the input of the variable stiffness module by screws. The slider moves along the linear guide rail, and the deformation of the linear spring changes. The model of the linear guide rail and slider is C-LUBE ML3 of IKO Company. The employment of the linear guide rail and slider can reduce friction and provide directional guidance for changing the deformation of the spring.

One end of the lever is clamped to the force point on the output of the variable stiffness module by a retainer ring. The design of the double-deck grooves in the lever allows the pivot to move freely in the groove, avoiding the geometric constraint between the force point or spring point and pivot. Consequently, the stiffness of the variable stiffness module can change from zero to infinity. The motor base is connected to the stiffness motor and the input of the variable stiffness module by screws. The stiffness motor shaft is connected to the wave generator of the harmonic reducer through the connector, and the flex spline and circular spline of the harmonic reducer are connected to the motor base and the Archimedean spiral cam by screws, respectively. When the stiffness motor is working, the Archimedean spiral cam rotates relative to the motor base (the input of the variable stiffness module), and the pivots move along the linear grooves on the input of the variable stiffness module. Two ends of the linear groove are located at the midpoint of the spring axis and the force point, restricting the movement of the pivots in the range of $0-18 \mathrm{~mm}$. A brushless DC flat motor EC-45 of Maxon Company with a rated power of $50 \mathrm{~W}$ is selected as the stiffness motor. Moreover, the stiffness motor integrates the MILE incremental encoder that can be utilized to measure the pivot position by calculation. A cup type harmonic reducer CSD-14-100-2A-R of Harmonic Drive Company is selected to transfer the motion of the stiffness motor.

Two pairs of ultrathin section bearings S08003CS0 and S07403CS0 of Kaydon Company are selected to reduce the motion friction between the output of the variable stiffness module or the motor base and the input of the variable stiffness module. The employment of ultrathin section bearings not only effectively reduces the axial size and makes the variable stiffness module more compact but also vacates the radial space in the variable stiffness module, which is conducive to the arrangement of other parts.

\subsection{Electrical system design of the VSA}

An expandable electrical system is proposed in this paper, and the framework of the electrical system is shown in Fig. 10. The dashed part represents other VSAs that can be employed together to build a manipulator. The electrical system employs a distributed structure including a central controller and an actuator controller. The central controller is mainly responsible for motion planning of the VSA or manipulator, operation of the control algorithm, communication with the host computer, and other upper tasks. Sensor information acquisition and processing, brake and motor control, and other underlying tasks are executed by the actuator controller. This distributed structure of the electrical system has two specific advantages: 1) It provides convenience for the construction of the VSA manipulator in the future; 2) compared with a single controller, the calculation burden of the controller is relatively low, and the electrical system is easy to maintain. 
Figure 11(a) shows that the physical hardware of the central controller is a self-developed digital signal processor/field programmable gate array (DSP/FPGA) control board based on compact peripheral component interconnect $(\mathrm{cPCI})$. The main processor of the $\mathrm{DSP} /$ FPGA control board is a TMS320C6000 series DSP of TI Company. To improve the speed and reliability of data communication, the DSP/FPGA control board also integrates a communication module with FPGA as the main processor. This communication module implements point-to-point high speed serial communication protocol [46] proposed by our laboratory for the communication between the central controller and the actuator controller. On the DSP/FPGA control board, the signals between FPGA and DSP are transmitted via the parallel interface.

Figure 11(b) shows that the actuator controller incorporates a control board, a driving board, and a connecting board. The microprocessor in charge of the high-speed communication with the central controller and sensor information processing selects a Cyclone IV series FPGA of Altera Company. The drive board is mainly responsible for receiving commands from the control board and driving the motor and brake. The actuator controller is not integrated into the VSA but rather intended to be placed inside the link of the manipulator in the future for reducing the volume of the VSA.

\section{Experiments and results}

To assess the performance of the symmetrical pivot VSA designed, experiments of stiffness calibration, stiffness motor response, and tracking are carried out in this section.

\subsection{Stiffness calibration experiment}

To calibrate the stiffness of the VSA, an experimental platform for stiffness calibration is built, as shown in Fig. 12. The output of the VSA is connected to a loading disc. The two circumferential directions of the loading disc are attached to the sliced weights by Kevlar ropes via pulleys.

The stiffness of the VSA is calibrated by means of loading and unloading the weights. Flexible deformation angle $\varphi$ and elastic torque $\tau_{\mathrm{e}}$ are taken as the $x$ and $y$ coordinates, respectively, to plot the curves of the calibration results. Thus, the slope of the calibration curves reflect the stiffness of the VSA. The principal motor remains stationary, and only the position of the stiffness motor is changed. Flexible deformation angle $\varphi$

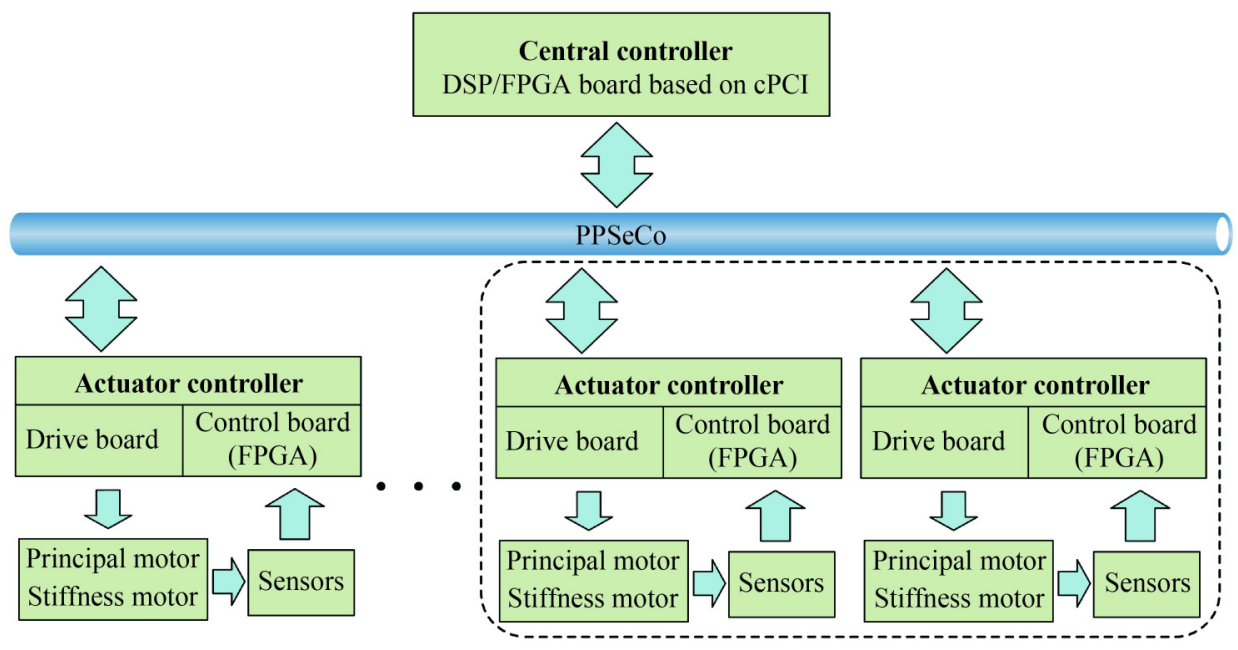

Fig. 10 Framework of the electrical system.

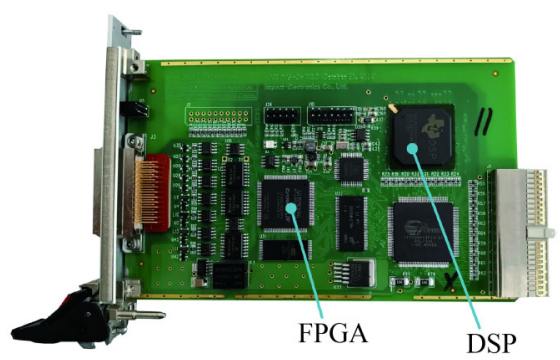

(a)

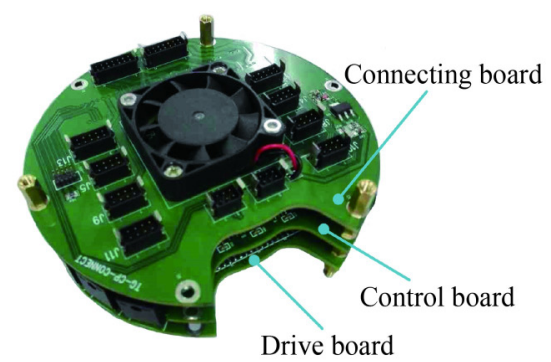

(b)

Fig. 11 Hardware of the controller: (a) central controller, (b) actuator controller. 
can be calculated as Eq. (19) because two magnetic encoders equipped by the drive mechanism can measure principal motor position $\theta_{1}$ and link-side position $q$.

$$
\varphi=\theta_{1}-q .
$$

The mass of each weight is $2 \mathrm{~kg}$, and the diameter of the loading disc plate is $200 \mathrm{~mm}$. The elastic torque of the VSA can be calculated by multiplying the total weight of the loaded weights by the radius of the loading disc. Experiments are repeated with different stiffness motor positions of $10^{\circ}, 30^{\circ}, 50^{\circ}, 70^{\circ}$, and $90^{\circ}$, and the corresponding pivot positions are 16.6, 13.8, 11, 8.2, and $5.4 \mathrm{~mm}$, respectively. The calibration results are shown in Fig. 13. The spotted solid line is the actual loading curve, the solid triangular line represents the actual unloading curve, and the dashed line represents the theoretical curve obtained Eq. (9). Figure 13 shows that the shape of the actual curve is consistent with that of the theoretical curve: the slope of the curve, i.e., the stiffness of the VSA increases as the pivots approach the force point. The actual curves almost coincide with the theoretical curves if the pivots are close to the force point. As the pivots move away from the force point, the actual curves shift to the right of the theoretical curves and gradually move away from the theoretical curves. This phenomenon may be related to the accuracy of part machining and assembly. When the pivots are far from the force point, deviations between the loading curves and unloading curves are larger. In this case, the stiffness of the VSA

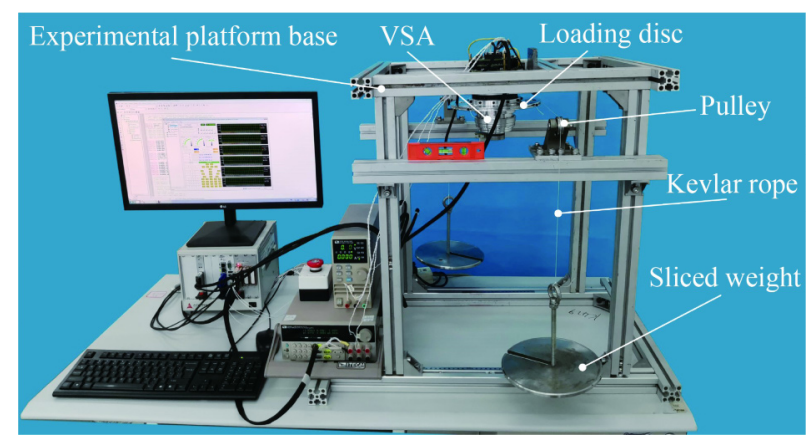

Fig. 12 Experimental platform for stiffness calibration.

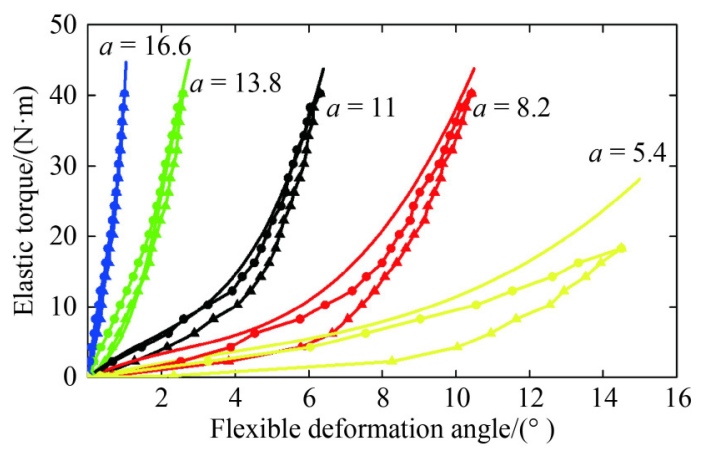

Fig. 13 Stiffness calibrate results of the VSA. shows an apparent hysteresis, which can be considered caused by the internal friction and linear spring backlash.

The deviation between the loading curve and the unloading curve is substantial when the position of the stiffness motor is $90^{\circ}$. The stiffness hysteresis of the VSA is so serious that determining the true value of the VSA stiffness is difficult. In this case, the minimum stiffness of the VSA is only approximately $15 \mathrm{~N} \cdot \mathrm{m} / \mathrm{rad}$, which shows a high compliance. Therefore, continuing to reduce VSA stiffness by adjusting the position of the stiffness motor is not meaningful. Moreover, if the VSA stiffness continues to decrease, the output of the VSA cannot return to the original position due to the internal friction after the load force disappears. Thus, the effective working range of the stiffness motor is from $0^{\circ}$ to $90^{\circ}$.

\subsection{Stiffness motor response experiment}

Stiffness adjustment time is an important performance indicator of the VSA. The shorter the stiffness adjustment time is, the better dynamic performance of the stiffness adjustment. Having fast, stable characteristics of stiffness adjustment is more necessary for VSA in performing explosive tasks. An experimental platform as shown in Fig. 14 is built for stiffness response experiment, together with the tracking experiments in next section. The coordinate system is established, as shown in Fig. 14. The rotational center of the VSA, the horizontal right direction, and the vertical upward direction are taken as the coordinate origin, the positive direction of the $x$ axis, and the positive direction of the $y$ axis, respectively. Thus, the angle between the link of the VSA and the positive direction of the $x$ axis is link-side position $q$.

According to the geometric parameters of the variable stiffness module, the Archimedean spiral cam can rotate $128.57^{\circ}$ relative to the input of the variable stiffness module as the pivot moves from one end of the linear

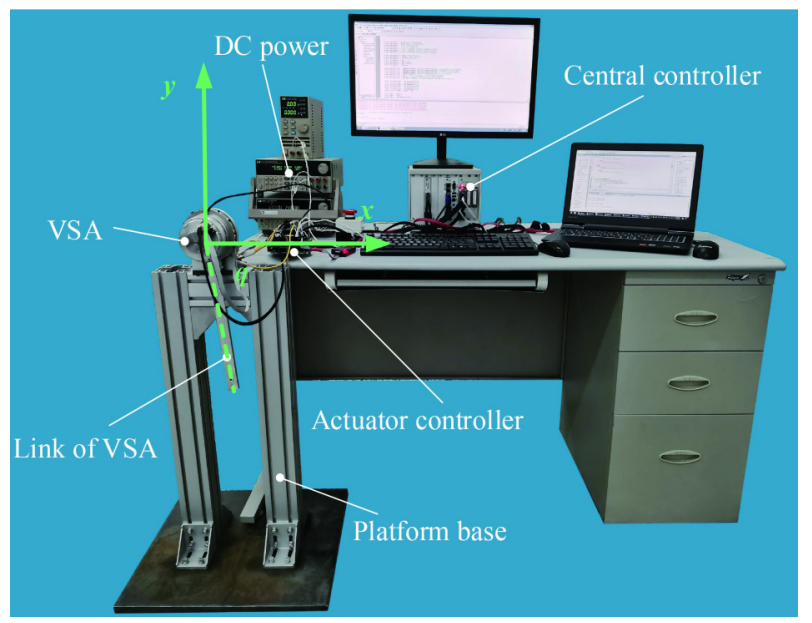

Fig. 14 Experimental platform for stiffness motor response and tracking. 
groove to the other. The proportional-derivative control with feedforward compensation [47] is employed in the position control of the stiffness motor. The controller of the stiffness motor position $\theta_{2}$ is given by

$$
u_{2}=-k_{2 \mathrm{p}} e_{2}-k_{2 \mathrm{~d}} \dot{e}_{2}+\tau_{\mathrm{r}}
$$

where $u_{2}$ is the control command of the stiffness motor, $e_{2}=\theta_{2}-\theta_{2 \mathrm{~d}}$ is the position error, and $\theta_{2 \mathrm{~d}}$ is the desired position for the stiffness motor, and $k_{2 \mathrm{p}}$ and $k_{2 \mathrm{~d}}$ are the proportional and derivative coefficients of control command $u_{2}$, respectively.

Step responses for the stiffness motor are conducted to adjust the stiffness of the VSA from infinity to zero and from zero to infinity at different loads $(0$ and $5 \mathrm{~kg})$. When the pivot is located at the force point and the corresponding stiffness of the VSA is positive infinity, stiffness motor position $\theta_{2}$ is set as zero. To show the performance of the closed-loop control strategy of the stiffness motor, the desired positions of the stiffness motor are set as $125^{\circ}$ (stiffness of VSA is close to zero) and $3^{\circ}$ (stiffness of VSA is close to infinity) when the initial positions of the stiffness motor are $0^{\circ}$ and $128.57^{\circ}$, respectively. Principal motor position $\theta_{1}$ is maintained at zero so that the load can apply torque to the stiffness motor to the most extent.

Figure 15 shows that regardless of adjusting the stiffness of the VSA from infinity to zero or from zero to infinity, the position of the stiffness motor can track the desired position without overshoot in the zero load condition, whereas the $5 \mathrm{~kg}$ load condition leads to a small overshoot, under the same control coefficients. Furthermore, the position step responses take approximately $0.4 \mathrm{~s}$ to adjust the position of the stiffness motor from $0^{\circ}$ to $125^{\circ}$ or from $128.57^{\circ}$ to $3^{\circ}$ in all conditions, which lays a hardware foundation for quickly responding to the stiffness adjustment of the VSA.

\subsection{Tracking experiment}

Tracking quality can demonstrate the dynamic perfor-

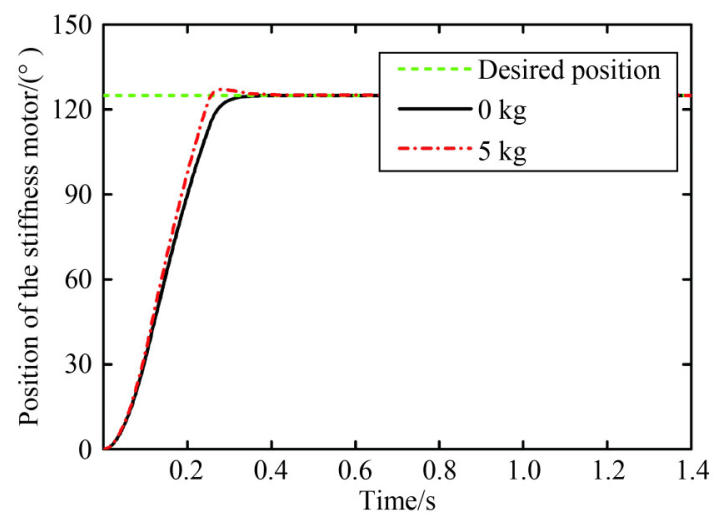

(a) mance of the manipulator or the actuator. The controller for robots with elastic joints proposed in Ref. [48] is employed to realize the trajectory tracking of the designed VSA. The controller of link position $q$ is given by

$$
u_{1}=-k_{1 \mathrm{p}} e_{1}-k_{1 \mathrm{~d}} \dot{e}_{1}+g\left(q_{\mathrm{d}}\right),
$$

where $u_{1}$ is the control command of the principal motor, $q_{\mathrm{d}}$ is the desired position for link, $g$ is the gravity term, $k_{1 \mathrm{p}}$ and $k_{1 \mathrm{~d}}$ are the proportional and derivative coefficients of control command $u_{1}$, respectively, $e_{1}=\theta_{1}-\theta_{1 \mathrm{~d}}$ is the position error, and $\theta_{1 \mathrm{~d}}$ is the desired position for principal motor.

Desired position $\theta_{1 \mathrm{~d}}$ can be given by

$$
\theta_{1 \mathrm{~d}}=q_{\mathrm{d}}+K^{-1} g\left(q_{\mathrm{d}}\right) \text {. }
$$

Sinusoidal tracking experiments in three cases are conducted to assess the tracking performance of the VSA. Figure 16(a) shows the position tracking performance at $1 /(2 \pi) \mathrm{Hz}$ with different loads ( 1 and $5 \mathrm{~kg}$ ). Pivot position $a$ is set as $12 \mathrm{~mm}$, which leads to a relatively high stiffness of the VSA. Figure 16(a) shows that both trajectories closely match the corresponding desired references, but the tracking trajectory exhibits a higher accuracy in the $1 \mathrm{~kg}$ load condition than in the $5 \mathrm{~kg}$ load condition. This finding indicates that tracking performance is degraded as load increases.

Figure 16(b) shows the position tracking performance at the same reference trajectory and with the same load (5 kg) but different pivot positions (different stiffnesses). The low stiffness considerably decreases the position tracking accuracy. The lower stiffness of the VSA further pulls down the system bandwidth. Figure 16(c) shows the position tracking performance at $1 /(2 \pi)$ and $1 / \pi \mathrm{Hz}$ with the same load $(5 \mathrm{~kg})$ and the same pivot position (same stiffness). Figure 16(c) shows that trajectory tracking performance deteriorates, and delay grows as reference frequency increases.

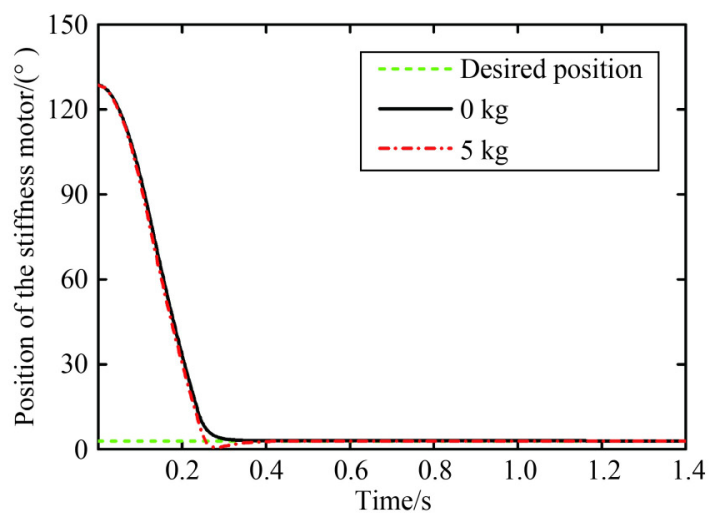

(b)

Fig. 15 Step response for stiffness motor: (a) adjusting stiffness from infinity to zero, (b) adjusting stiffness from zero to infinity. 


\section{Discussion}

A novel VSA with a modular design is presented. One innovation of the designed VSA is that the stiffness can be adjusted from zero to infinity by virtue of the doubledeck grooves design in the lever compared with other VSAs based on the lever principle with a variable pivot such as vsaUT-II and SVSA. Another innovation of the designed VSA is the symmetrical stiffness adjustment mechanism that is used to change the positions of a pair of pivots. To our knowledge, SVSA-II is the only actuator with a symmetric structure based on the variable pivot lever principle other than the VSA designed in this paper. Other VSAs, for example vsaUT-II, CompActVSA, AwAS-II, and SVSA, have an asymmetric structure of stiffness adjustment mechanism that may cause stress concentration, bring additional bending moment, and decrease mechanical reliability. Although the VSA designed in this paper also changes the pivot position by means of the Archimedean spiral relocation principle such as SVSA-II, the Archimedean spiral cam in the designed VSA is directly driven by a harmonic reducer instead of a gear system, which can change pivot position without backlash. Other VSAs based on the lever principle with a variable pivot such as CompAct-VSA,
vsaUT-II, and SVSA also drive the pivot position through the gear system. Furthermore, the modular design proposed in this paper but lost in other VSAs enables the rigid actuator to become VSA by mounting the designed variable stiffness module, which is the most important innovation of this paper.

To verify the superiority of the proposed design further, comparisons with well-known VSAs are listed in Table 1. Table 1 shows that only a few VSAs based on the lever principle with a variable pivot have a stiffness adjustment range from zero to infinity. Although not fast, the stiffness regulation speed of the designed VSA ranks high among all the designs. In terms of size and mass, the designed VSA only has a middle rank among all the designs because it contains a brake mechanism. Its size and mass can be greatly reduced if the brake mechanism is removed. Furthermore, it can cover a large range of motion $\left(0^{\circ}-360^{\circ}\right)$ and has a high driving capacity (nominal torque is $36 \mathrm{~N} \cdot \mathrm{m}$, and peak torque is $68 \mathrm{~N} \cdot \mathrm{m}$ ). The output of the designed VSA is a cylinder with a large diameter rather than a thin rod such as SVSA-II, CompAct-VSA, and other VSAs. These characteristics allow the designed VSA to satisfy the requirements of real robotic applications.

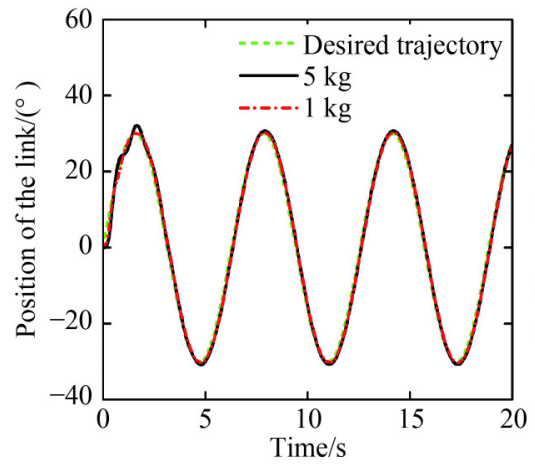

(a)

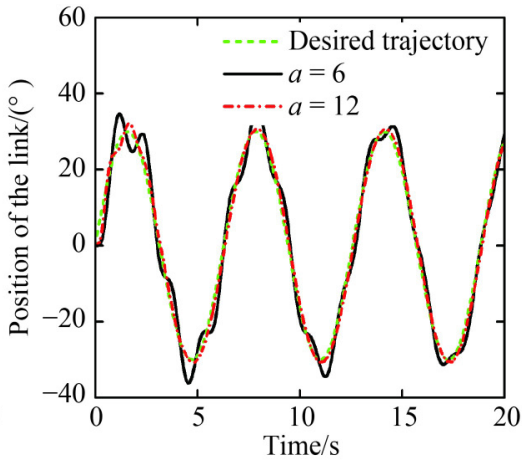

(b)

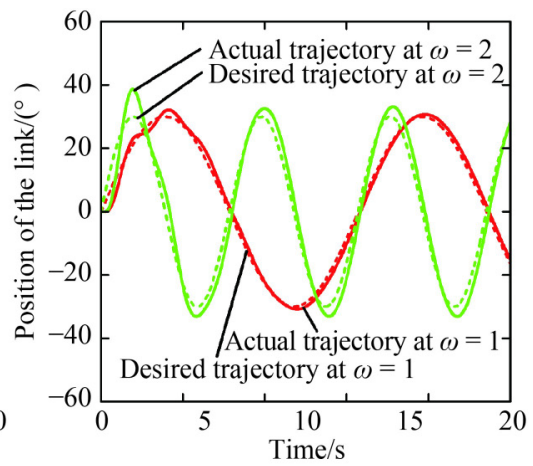

(c)

Fig. 16 Tracking experiments: (a) different loads, (b) different stiffnesses, and (c) different frequencies.

Table 1 Comparisons of different VSAs

\begin{tabular}{|c|c|c|c|c|c|c|c|c|c|}
\hline Name & $\mathrm{RoS} /\left(\mathrm{N} \cdot \mathrm{m} \cdot \mathrm{rad}^{-1}\right)$ & $\mathrm{RST} / \mathrm{s}$ & $\operatorname{RoM} /\left(^{\circ}\right)$ & $\mathrm{NT} /(\mathrm{N} \cdot \mathrm{m})$ & $\mathrm{PT} /(\mathrm{N} \cdot \mathrm{m})$ & Mass $/ \mathrm{kg}$ & $\mathrm{Size} /(\mathrm{mm} \times \mathrm{mm})$ & Modular & Brake \\
\hline FSJ [29] & $52.4-826$ & 0.33 & $0-180$ & 31.3 & 67 & 1.41 & $94 \times 118$ & No & No \\
\hline MACCEPA [27] & $5-110$ & 10.00 & $0-300$ & 40 & 70 & 2.4 & I & No & No \\
\hline vsaUT-II [34] & $0.7-948$ & 0.90 & $0-57.2$ & 21.8 & 60 & 2.5 & 1 & No & No \\
\hline$S^{3}$ VSA [30] & $12.12-\infty$ & 0.10 & $0-360$ & 7.6 & 22.7 & 1.54 & $118 \times 125$ & No & No \\
\hline CompAct-VSA [36] & $0-\infty$ & 0.10 & $0-360$ & / & 117 & 1 & $120 \times 160$ & No & No \\
\hline AwAS-II [37] & $0-\infty$ & 5.00 & $0-165$ & / & 80 & 1.1 & $140 \times 180$ & No & No \\
\hline SVSA [31] & $1.7-150.56$ & $0.60^{*}$ & $0-360$ & 9.46 & 22.1 & 2.4 & l & No & No \\
\hline SVSA-II [41] & $0-\infty$ & $0.30 *$ & $0-360$ & / & 25 & 1.647 & l & No & No \\
\hline Proposed VSA & $0-\infty$ & 0.40 & 0-360 & 36 & 68 & 2.46 & $104 \times 156$ & Yes & Yes \\
\hline
\end{tabular}

* Values estimated from literature, RoS: range of stiffness, SRT: stiffness regulation time, RoM: range of motion, NT: nominal torque, PT: peak torque. 


\section{Conclusions}

This paper designs a novel VSA with a symmetrical pivot adjustment. The stiffness adjustment scheme based on the symmetrical variable pivot principle that can eliminate the additional bending moment caused by the asymmetric structure is proposed, and the characteristics of this symmetrical stiffness adjustment scheme are analyzed systematically. Based on this scheme, the symmetrical stiffness adjustment mechanism is designed to change the position of a pair of pivots with high synchronization and easy control. Transferring motion in a roundabout manner presented in this paper realizes the modular design of the VSA, including drive module and variable stiffness module. An expandable electrical system with a distributed structure that will facilitate the construction of a manipulator in the future is also introduced. The VSA designed has the advantages of compact structure, high loading capacity, large stiffness adjustment range, and fast stiffness adjustment speed. Future study will focus on the stiffness identification online that can accurately estimate the stiffness of the designed VSA and investigate the implementation of torque control for the VSA and building a manipulator with the designed VSA.

\section{Nomenclature}

\section{Abbreviations}

$\begin{array}{ll}\text { cPCI } & \text { Compact peripheral component interconnect } \\ \text { VSA } & \text { Variable stiffness actuator } \\ \text { DSP } & \text { Digital signal processor } \\ \text { FPGA } & \text { Field programmable gate array } \\ \text { HIT } & \text { Harbin Institute of Technology, China }\end{array}$

Variables

a Vertical distance from the pivot to the spring axis

A Angle between the lever and the output link

$b \quad$ Distance between the spring axis and the symmetrical center line of the variable stiffness module

c Polar radius per polar angle

d Polar radius when $\rho=0$

$e_{1}, e_{2} \quad$ Position error, $e_{1}=\theta_{1}-\theta_{1 \mathrm{~d}}, e_{2}=\theta_{2}-\theta_{2 \mathrm{~d}}$

E Stored elastic energy

$f_{\mathrm{p}} \quad$ Force applied by the pivot on the Archimedean spiral cam, $f_{\mathrm{p}}=-F_{\mathrm{p}}$

$f_{\mathrm{pa}} \quad$ Decomposed of $f_{\mathrm{p}}$ along the direction perpendicular to the spring axis

$f_{\text {pat }} \quad$ Decomposed of $f_{\mathrm{pa}}$ along the direction perpendicular to the tangent of the Archimedean spiral line
$F_{\mathrm{e}} \quad$ Elastic force of the VSA generated at the force point in the opposite direction of load force $F_{1}$

$F_{1} \quad$ Load force applied at the force point

$F_{\text {lt }} \quad$ Decomposed along the direction perpendicular to the lever

$F_{\mathrm{p}} \quad$ Force applied on the lever from the pivot

$F_{\mathrm{pa}} \quad$ Decomposed along the direction perpendicular to the spring axis

$F_{\mathrm{S}} \quad$ Spring force

$F_{\text {st }} \quad$ Decomposed along the direction perpendicular to the lever

$g \quad$ Gravity term

$k \quad$ Spring stiffness

$k_{1 \mathrm{p}}, k_{1 \mathrm{~d}} \quad$ Proportional and derivative coefficients of control command $u_{1}$, respectively

$k_{2 \mathrm{p}}, k_{2 \mathrm{~d}} \quad$ Proportional and derivative coefficients of control command $u_{2}$, respectively

$K \quad$ Stiffness of the VSA

$l \quad$ Total length of the lever

$l_{1}, l_{2} \quad$ Distances from the pivot to the spring point and the force point, respectively

$q \quad$ Link-side position

$q_{\mathrm{d}} \quad$ Desired position for link

$r \quad$ Arm of the force $f_{\text {pat }}$ relative to the coordinate origin

$R \quad$ Total length of the output link, i.e., the distance between the force point and the center of the variable stiffness module

$S \quad$ Curvature of the Archimedean spiral line at $P(x, y)$

$S_{1} \quad$ Slope between point $P(x, y)$ and the coordinate origin

$u_{1}, u_{2} \quad$ Control commands of the principal and stiffness motors, respectively

$\Delta x \quad$ Deformation of the spring

$\beta \quad$ Angle between the force $f_{\mathrm{pa}}$ and the tangent of the Archimedean spiral line

$\gamma \quad$ Angle between the lever and the linear groove of the input link

$\tau_{1} \quad$ Load torque exerted on the output link

$\tau_{\mathrm{e}} \quad$ Elastic torque of the variable stiffness module

$\tau_{\mathrm{r}} \quad$ Resistance torque

$\theta_{1}, \theta_{2} \quad$ Principal and stiffness motor positions, respectively

$\theta_{1 \mathrm{~d}}, \theta_{2 \mathrm{~d}}$ Desired positions for the principal and stiffness motors, respectively

$\varphi \quad$ Angle between the input link and the output link that represents the flexible deformation angle of the VSA

$\rho \quad$ Polar angle of point $P(x, y)$ on the Archimedean spiral line

Acknowledgement This work was supported by the National Key R\&D Program of China (Grant No. 2017YFB1300400) and the National Natural Science Foundation of China (Grant No. 51805107).

Open Access This article is licensed under a Creative Commons Attribution 4.0 International License, which permits use, sharing, adaptation, distribution, and reproduction in any medium or format as long as appropriate credit is given to the original author(s) and source, a link to the Creative Commons license is provided, and the changes made are indicated. 
The images or other third-party material in this article are included in the article's Creative Commons license, unless indicated otherwise in a credit line to the material. If material is not included in the article's Creative Commons license and your intended use is not permitted by statutory regulation or exceeds the permitted use, you will need to obtain permission directly from the copyright holder.

To view a copy of this license, visit http://creativecommons.org/licenses/ by $/ 4.0 \%$.

\section{References}

1. Ren Y, Chen Z, Liu Y, et al. Adaptive hybrid position/force control of dual-arm cooperative manipulators with uncertain dynamics and closed-chain kinematics. Journal of the Franklin Institute, 2017, 354(17): 7767-7793

2. Perrusquía A, Yu W, Soria A. Position/force control of robot manipulators using reinforcement learning. Industrial Robot, 2019, 46(2): 267-280

3. $\mathrm{Su} \mathrm{T}$, Niu L, He G, et al. Coordinated variable impedance control for multi-segment cable-driven continuum manipulators. Mechanism and Machine Theory, 2020, 153: 103969

4. Ott C, Mukherjee R, Nakamura Y. Unified impedance and admittance control. In: Proceedings of IEEE International Conference on Robotics and Automation. Anchorage: IEEE, 2010, $554-561$

5. Albu-Schäffer A, Ott C, Hirzinger G. A unified passivity based control framework for position, torque and impedance control of flexible joint robots. International Journal of Robotics Research, 2007, 26(1): 5-21

6. Wang N, Chen B, Ge X, et al. Modular crawling robots using soft pneumatic actuators. Frontiers of Mechanical Engineering, 2021, 16(1): 163-175

7. Korayem M H, Rahimi H N. Nonlinear dynamic analysis for elastic robotic arms. Frontiers of Mechanical Engineering, 2011, 6(2): 219-228

8. Chiaradia D, Tiseni L, Frisoli A. Compact series visco-elastic joint (SVEJ) for smooth torque control. IEEE Transactions on Haptics, 2020, 13(1): 226-232

9. Stuhlenmiller F, Clos D, Rinderknecht S, et al. Impact of friction and gait parameters on the optimization of series elastic actuators for gait assistance. Mechanism and Machine Theory, 2019, 133: 737-749

10. Wang $\mathrm{P}, \mathrm{Zhu} \mathrm{Q}, \mathrm{Hu} \mathrm{X}$, et al. Research on interaction safety of human-robot collision based on series elastic actuator. In: Proceedings of the 5th International Conference on Information, Cybernetics, and Computational Social Systems. Hangzhou: IEEE, 2018, 180-185

11. Liu Y, Wang D, Yang S, et al. Design and experimental study of a passive power-source-free stiffness-self-adjustable mechanism. Frontiers of Mechanical Engineering, 2021, 16(1): 32-45

12. Jiang $\mathrm{P}$, Yang $\mathrm{Y}$, Chen $\mathrm{M} \mathrm{Z}$, et al. A variable stiffness gripper based on differential drive particle jamming. Bioinspiration \& Biomimetics, 2019, 14(3): 036009

13. Liu L, Leonhardt S, Misgeld B J. Design and control of a mechanical rotary variable impedance actuator. Mechatronics,
2016, 39: 226-236

14. Pratt G A, Williamson M. Series elastic actuators. In: Proceedings of IEEE International Conference on Intelligent Robots and Systems. Pittsburgh: IEEE, 1995, 399-406

15. Cummings J P, Ruiken D, Wilkinson E L, et al. A compact, modular series elastic actuator. Journal of Mechanisms and Robotics, 2016, 8(4): 041016

16. Santos W M, Caurin G A, Siqueira A A. Design and control of an active knee orthosis driven by a rotary series elastic actuator. Control Engineering Practice, 2017, 58: 307-318

17. Braun D, Howard M, Vijayakumar S. Optimal variable stiffness control: formulation and application to explosive movement tasks. Autonomous Robots, 2012, 33(3): 1-17

18. Braun D J, Chalvet V, Chong T, et al. Variable stiffness spring actuators for low-energy-cost human augmentation. IEEE Transactions on Robotics, 2019, 35(6): 1435-1449

19. Vanderborght B, Verrelst B, Van Ham R, et al. Exploiting natural dynamics to reduce energy consumption by controlling the compliance of soft actuators. International Journal of Robotics Research, 2006, 25(4): 343-358

20. Roozing W, Li Z, Medrano C. Development and control of a compliant asymmetric antagonistic actuator for energy efficient mobility. IEEE/ASME Transactions on Mechatronics, 2015, 21(2): 1080-1091

21. Visser L C, Carloni R, Ünal R, et al. Modeling and design of energy efficient variable stiffness actuators. In: Proceedings of IEEE International Conference on Robotics and Automation. Anchorage: IEEE, 2010, 3273-3278

22. Shao Y, Zhang W, Su Y, et al. Design and optimisation of loadadaptive actuator with variable stiffness for compact ankle exoskeleton. Mechanism and Machine Theory, 2021, 161: 104323

23. Liu Y, Liu X, Yuan Z, et al. Design and analysis of spring parallel variable stiffness actuator based on antagonistic principle. Mechanism and Machine Theory, 2019, 140: 44-58

24. Guo J, Tian G. Mechanical design and analysis of the novel 6-DOF variable stiffness robot arm based on antagonistic driven joints. Journal of Intelligent \& Robotic Systems, 2016, 82(2): 207-235

25. Petit F, Friedl W, Höppner H, et al. Analysis and synthesis of the bidirectional antagonistic variable stiffness mechanism. IEEE/ASME Transactions on Mechatronics, 2015, 20(2): 684-695

26. Friedl W, Höppner H, Petit F, et al. Wrist and forearm rotation of the DLR Hand Arm System: Mechanical design, shape analysis and experimental validation. In: Proceedings of IEEE/RSJ International Conference on Intelligent Robots and Systems. San Francisco: IEEE, 2011, 1836-1842

27. Vanderborght B, Tsagarakis N, Semini C, et al. MACCEPA 2.0: adjustable compliant actuator with stiffening characteristic for energy efficient hopping. In: Proceedings of IEEE International Conference on Robotics and Automation. Kobe: IEEE, 2009, 544-549

28. Wolf S, Hirzinger G. A new variable stiffness design: matching requirements of the next robot generation. In: Proceedings of IEEE International Conference on Robotics and Automation. Pasadena: 2008, 1741-1746

29. Wolf S, Eiberger O, Hirzinger G. The DLR FSJ: energy based design of a variable stiffness joint. In: Proceedings of IEEE 
International Conference on Robotics and Automation. Shanghai: IEEE, 2011, 5082-5089

30. Xu Y, Guo K, Li J, et al. A novel rotational actuator with variable stiffness using S-shaped springs. IEEE/ASME Transactions on Mechatronics, 2021, 26(4): 2249-2260

31. Sun J, Guo Z, Zhang Y, et al. A novel design of serial variable stiffness actuator based on an Archimedean spiral relocation mechanism. IEEE/ASME Transactions on Mechatronics, 2018, 23(5): 2121-2131

32. Sun J, Zhang Y, Zhang C, et al. Mechanical design of a compact serial variable stiffness actuator (SVSA) based on lever mechanism. In: Proceedings of IEEE International Conference on Robotics and Automation. Singapore: IEEE, 2017, 33-38

33. Barrett E, Fumagalli M, Carloni R. Elastic energy storage in leaf springs for a lever-arm based variable stiffness actuator. In: Proceedings of IEEE/RSJ International Conference on Intelligent Robots and Systems. Daejeon: IEEE, 2016, 537-542

34. Groothuis S, Rusticelli G, Zucchelli A, et al. The variable stiffness actuator vsaUT-II: mechanical design, modeling, and identification. IEEE/ASME Transactions on Mechatronics, 2014, 19(2): 589-597

35. Fumagalli M, Barrett E, Stramigioli S, et al. The mVSA-UT: a miniaturized differential mechanism for a continuous rotational variable stiffness actuator. In: Proceedings of the 4th IEEE RAS \& EMBS International Conference on Biomedical Robotics and Biomechatronics. Rome: IEEE, 2012, 1943-1948

36. Tsagarakis N, Sardellitti I, Caldwell D G. A new variable stiffness actuator (CompAct-VSA): design and modelling. In: Proceedings of IEEE/RSJ International Conference on Intelligent Robots and Systems. San Francisco: IEEE, 2011, 378-383

37. Jafari A, Tsagarakis N, Caldwell D G. AwAS-II: a new actuator with adjustable stiffness based on the novel principle of adaptable pivot point and variable lever ratio. In: Proceedings of IEEE International Conference on Robotics and Automation. Shanghai: IEEE, 2011, 4638-4643

38. Visser L C, Carloni R, Stramigioli S. Energy-efficient variable stiffness actuators. IEEE Transactions on Robotics, 2011, 27(5):
865-875

39. Rao S, Carloni R, Stramigioli S. A novel energy-efficient rotational variable stiffness actuator. In: Proceedings of Annual International Conference of the IEEE Engineering in Medicine and Biology Society. Boston: IEEE, 2011, 8175-8178

40. Visser L C, Carloni R, Stramigioli S. Variable stiffness actuators: a port-based analysis and a comparison of energy efficiency. In: Proceedings of IEEE International Conference on Robotics and Automation. Anchorage: IEEE, 2010, 3279-3284

41. Sun J, Guo Z, Sun D, et al. Design, modeling and control of a novel compact, energy-efficient, and rotational serial variable stiffness actuator (SVSA-II). Mechanism and Machine Theory, 2018, 130: 123-136

42. Wu J, Wang Z, Chen W, et al. Design and validation of a novel leaf spring-based variable stiffness joint with reconfigurability. IEEE/ASME Transactions on Mechatronics, 2020, 25(4): 2045-2053

43. Shao Y, Zhang W, Ding X. Configuration synthesis of variable stiffness mechanisms based on guide-bar mechanisms with lengthadjustable links. Mechanism and Machine Theory, 2021, 156: 104153

44. Zhang Z, Ni F, Dong Y, et al. A novel absolute angular position sensor based on electromagnetism. Sensors and Actuators. A, Physical, 2013, 194: 196-203

45. Zhang Z, Ni F, Dong Y, et al. A novel absolute magnetic rotary sensor. IEEE Transactions on Industrial Electronics, 2015, 62(7): 4408-4419

46. Liu H, Meusel P, Seitz N, et al. The modular multisensory DLRHIT-Hand. Mechanism and Machine Theory, 2007, 42(5): 612-625

47. Santibañez V, Kelly R. PD control with feedforward compensation for robot manipulators: analysis and experimentation. Robotica, 2001, 19(1): 11-19

48. Tomei P. A simple PD controller for robots with elastic joints. IEEE Transactions on Automatic Control, 1991, 36(10): $1208-1213$ 